\title{
Direct imaging of Beta Pictoris b with first-light Magellan Adaptive Optics
}

\author{
Katie M. Morzinski ${ }^{1, \ddagger} \dagger$, Laird M. Close ${ }^{1}$, Jared R. Males ${ }^{1, \ddagger}$, \\ Phil M. Hinz ${ }^{1}$, Alfio Puglisi ${ }^{2}$, Simone Esposito ${ }^{2}$, Armando Riccardi ${ }^{2}$, \\ Enrico Pinna ${ }^{2}$, Marco Xompero ${ }^{2}$, Runa Briguglio ${ }^{2}$, Kate Follette ${ }^{1}$, \\ Derek Kopon $^{3}$, Victor Gasho ${ }^{1}$, Alan Uomoto ${ }^{4}$, Tyson Hare ${ }^{4}$, \\ Andy Skemer ${ }^{1}$, Carmelo Arcidiacono ${ }^{2}$, Fernando Quiros-Pacheco ${ }^{2}$, \\ Javier Argomedo ${ }^{2}$, Lorenzo Busoni ${ }^{2}$, T. J. Rodigas ${ }^{1}$ and Ya-Lin $\mathbf{W u}^{1}$ \\ ${ }^{\ddagger}$ NASA Sagan Fellow \\ ${ }^{1}$ Steward Observatory, Center for Astronomical Adaptive Optics, University of Arizona, \\ 933 N. Cherry Ave., Tucson, AZ 85721, USA \\ ${ }^{2}$ Istituto Nazionale di Astrofisica, Osservatorio Astrofisico di Arcetri, Largo E Fermi 5, 50125 \\ Firenze, Italy \\ ${ }^{3}$ Max-Planck-Institut für Astronomie, Königstuhl 17, D-69117 Heidelberg, Germany \\ ${ }^{4}$ Carnegie Observatories, OCIW, 813 Santa Barbara St. Pasadena, CA 91101, USA
}

\begin{abstract}
MagAO is the newly-commissioned adaptive optics (AO) instrument on the Magellan Clay telescope at Las Companas Observatory, Chile. MagAO has two co-mounted science cameras: VisAO for visible-light direct and spectral-differential imaging; and Clio for near to thermal IR direct imaging, non-redundant-mask interference, and prism spectroscopy. We demonstrate MagAO's simultaneous visible and infrared AO performance via direct images of exoplanet Beta Pictoris b. The planet was detected in 5 passbands from $0.9-5 \mu \mathrm{m}$. Here we show the infrared images; the visible observations are presented in Males et al. 2013. MagAO is the first AO system to offer good performance with extensive coverage across the O/IR spectrum and thus offers an unprecedented opportunity to study the spectral energy distributions of directly-imaged extrasolar planetary atmospheres.
\end{abstract}

Keywords. Planets and satellites: individual (Beta Pictoris b); Stars: individual (Beta Pictoris); Stars: planetary systems; Instrumentation: adaptive optics

\section{Introduction}

The young nearby A-star $\beta$ Pictoris hosts an extensive edge-on disk discovered with optical imaging in the 1980s (Smith \& Terrile 1984). A massive planet was suspected to be the cause of the inner warp of the disk, but the companion was not confirmed until the direct imaging discovery by Lagrange et al. $(2009,2010)$. The planetary companion, $\beta$ Pic b, has been imaged with VLT/NaCo and Gemini-S/NICI at $J, H, K_{s},[4.05] \mu \mathrm{m}, L^{\prime}$, and $M^{\prime}$ (Bonnefoy et al. 2011, Boccaletti et al. 2013, Bonnefoy et al. 2013). We target this object for first-light demonstration of our new adaptive optics (AO) instrument, MagAO (Close et al. 2012).

MagAO is a 585-degree-of-freedom AO system on the Magellan Clay telescope at Las Campanas Observatory in Chile. Its features include a 378-mode modulating pyramid wavefront-sensor giving excellent performance, and an adaptive secondary mirror (ASM) giving improved thermal throughput out to $5 \mu \mathrm{m}$. MagAO has two science cameras that

\section{$\dagger$ email: ktmorz@arizona.edu}


can operate simultaneously for twice the observing efficiency: VisAO with broad and narrow passbands from $r^{\prime}$ to $Y_{s}$; and Clio with broad and narrow passbands from $J$ to $M^{\prime}$ (see http://magao.as.arizona.edu/observers/ for full information on the instrument). Here we show the performance of MagAO/Clio with first-light images of $\beta$ Pic b. These observations were carried out during the first commissioning run in NovemberDecember 2012.

\section{MagAO performance}

\subsection{PSF simulations}

We begin with a simulation of the MagAO/Clio PSF, for comparison to the on-sky performance. The major modifiers to the unitary amplitude of a circular pupil are (1) the secondary mirror obscuration and its support struts ("spiders"), and (2) the cold stop and its spiders within Clio's dewar. For the former, we used a digital camera image of the ASM taken while standing at the Nasmyth focus, prior to MagAO being mounted at the Nas. ring. For the latter, we use a Clio image of the cold stop taken in pupil imaging mode.

The composite of both images is the pupil image, shown in Fig. 1, left. The four thin arms are the ASM spiders and the two thick arms are the cold stop spiders. The lollipoplike shape at left (nine o'clock) is the "slot" cut in to the ASM surface to prevent a crack from spreading. The image at right shows the resultant PSF that we expect for the case of no wavefront error.
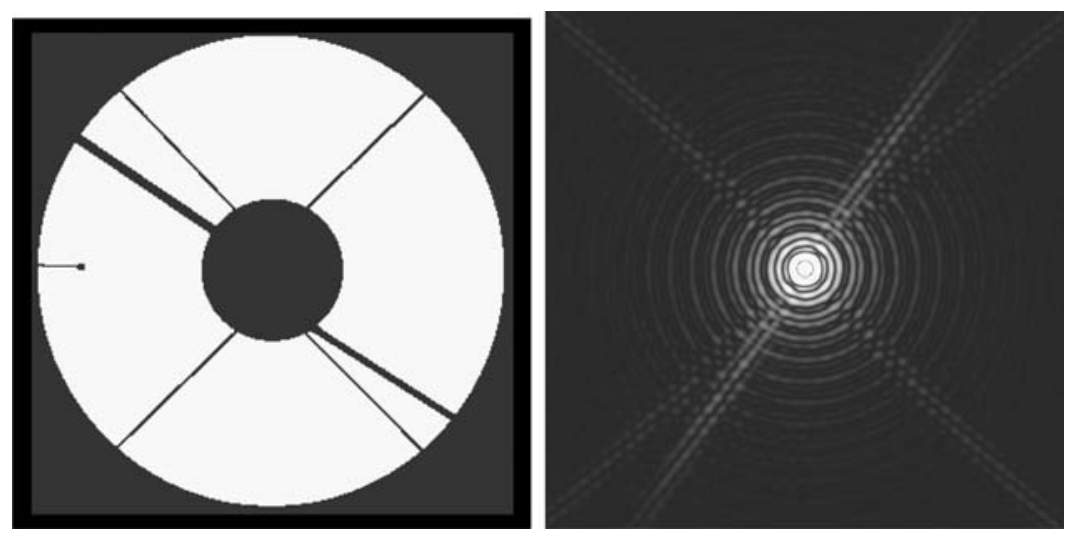

Figure 1. MagAO/Clio pupil and simulated PSF. A detailed discussion is available at http://visao.as arizona.edu/simulations/magao-pupils-and-fourier-optics/.

\subsection{On-sky PSFs}

Now that we have looked at the simulated PSF, we can compare the on-sky performance of MagAO/Clio. Figure 2 shows the $L^{\prime}$ and $M^{\prime}$ on-sky PSFs of $\beta$ Pic, as well as a comparison to the simulated PSF. The Airy pattern is clearly seen. What look like atmospheric speckes are actually the combination of diffraction spikes caused by spiders in the cold stop and ASM. There is no wavefront error in the simulated PSFs. This demonstrates the excellent high-order performance of MagAO. 

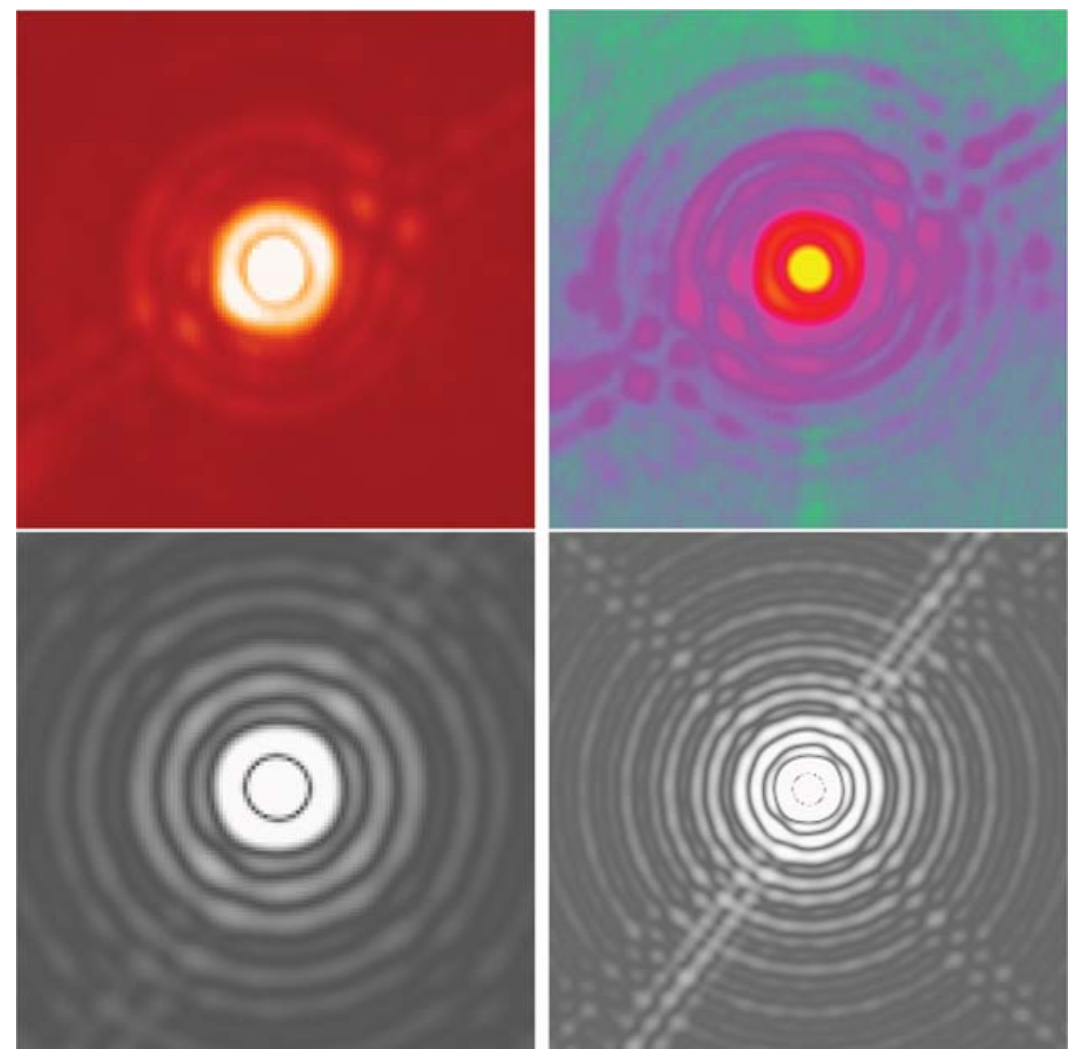

Figure 2. Top: $\beta$ Pic at $L^{\prime}$ (left) and $M^{\prime}$ (right). Bottom: Simulated PSF, varying zoom scales.

\section{Exoplanet images}

$\beta$ Pic was observed on five nights from 1-8 Dec. 2012 in five infrared filters from 2-5 $\mu \mathrm{m}$. We used pupil-tracking mode and reduced the data using angular differential imaging (ADI, Marois et al. 2006) and principal component analysis (PCA). The $L^{\prime}$ and $M^{\prime} \mathrm{PCA}$ residuals are shown in Fig. 3; the planet is clearly visible to the lower right (south west) of the star. $\beta$ Pic b is seen with high significance in all Clio passbands used. The mass of $\beta \mathrm{Pic} \mathrm{b}$ is estimated at $7-15 M_{\mathrm{Jup}}$ depending on the formation mechanism assumed (Bodenheimer et al. 2013). The MagAO images of the planet will help us to better determine its physical parameters, including mass and atmospheric composition. This demonstration illustrates the importance of high-order $\mathrm{O} / \mathrm{IR}$ adaptive optics for direct imaging of extrasolar planets.

\section{Summary}

Direct imaging of extrasolar planets allows us to carry out detailed characterization of planets beyond the snow line. Models are crucial to interpreting data, but at present more data is needed to constrain degeneracies. This first-light demonstration of MagAO/Clio has shown the AO instrument performance to be excellent quality, allowing us to image the planet $\beta \mathrm{Pic} \mathrm{b}$ in several thermal-infrared filters. MagAO offers unique capabilities to characterize directly-imaged exoplanets at $\mathrm{O} / \mathrm{IR}$ wavelengths from $0.55-5 \mu \mathrm{m}$. We refer potential MagAO/VisAO and MagAO/Clio users to http://magao.as.arizona.edu/ for web resources, SPIE publications, and guides for observers. 

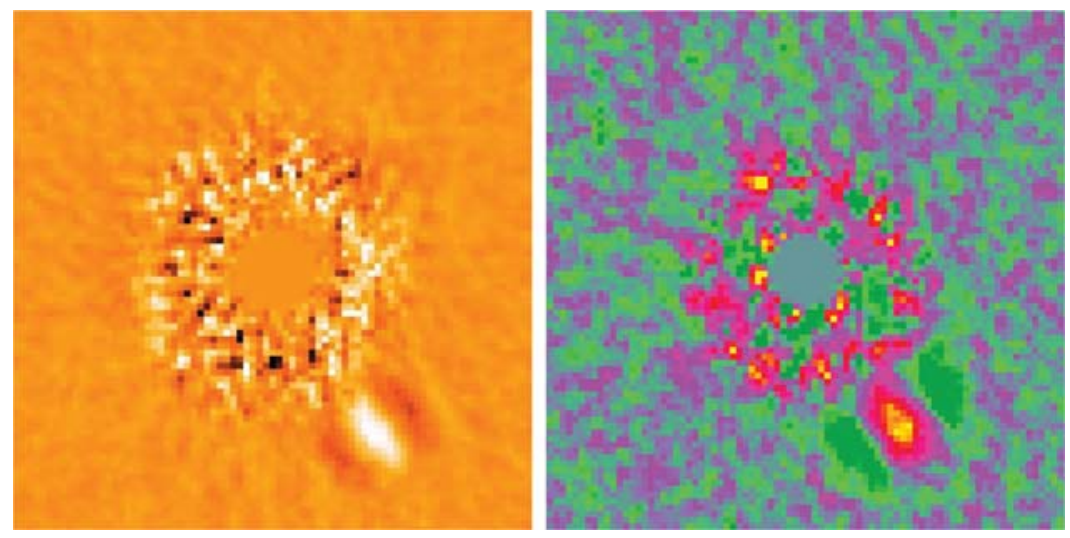

Figure 3. $\beta$ Pic b at $L^{\prime}$ (left) and $M^{\prime}$ (right).

\section{Acknowledgements}

The MagAO ASM was developed with support from the NSF MRI program. The MagAO PWFS was developed with help from the TSIP program and the Magellan partners. The Active Optics guider was developed by Carnegie Observatories with custom optics from the MagAO team. The VisAO camera and commissioning was supported with help from the NSF ATI program. The Clio camera comes courtesy of PMH and Steward Observatory. KMM was supported under contract with the California Institute of Technology (Caltech) funded by NASA through the Sagan Fellowship Program. LMCs research was supported by NSF AAG and NASA Origins of Solar Systems grants. JRM is grateful for the generous support of the Phoenix ARCS Foundation and is also newly supported through the Sagan Fellowship Program.

\section{References}

Boccaletti, A., Lagrange, A.-M., Bonnefoy, M., Galicher, R., \& Chauvin, G. 2013, A\& $A$, 551, L14

Bodenheimer, P., D’Angelo, G., Lissauer, J. J., Fortney, J. J., \& Saumon, D. 2013, ApJ, 770, 120

Bonnefoy, M., Boccaletti, A., Lagrange, A.-M., Allard, F., Mordasini, C., Beust, H., Chauvin, G., Girard, J. H. V., Homeier, D., Apai, D., Lacour, S., \& Rouan, D. 2013, A\&A A accepted

Bonnefoy, M., Lagrange, A.-M., Boccaletti, A., Chauvin, G., Apai, D., Allard, F., Ehrenreich, D., Girard, J. H. V., Mouillet, D., Rouan, D., Gratadour, D., \& Kasper, M. 2011, A\&\&A, $528, \mathrm{~L} 15$

Close, L. M., Males, J. R., Kopon, D. A., Gasho, V., Follette, K. B., Hinz, P., Morzinski, K., Uomoto, A., Hare, T., Riccardi, A., Esposito, S., Puglisi, A., Pinna, E., Busoni, L., Arcidiacono, C., Xompero, M., Briguglio, R., Quiros-Pacheco, F., \& Argomedo, J. 2012, Proc. SPIE, 8447, 84470X

Lagrange, A.-M., Bonnefoy, M., Chauvin, G., Apai, D., Ehrenreich, D., Boccaletti, A., Gratadour, D., Rouan, D., Mouillet, D., Lacour, S., \& Kasper, M. 2010, Science, 329, 57

Lagrange, A.-M., Gratadour, D., Chauvin, G., Fusco, T., Ehrenreich, D., Mouillet, D., Rousset, G., Rouan, D., Allard, F., Gendron, É., Charton, J., Mugnier, L., Rabou, P., Montri, J., \& Lacombe, F. 2009a, $A \mathscr{E} A$, 493, L21

Males, J. R., et al. 2013, this Volume

Marois, C., Lafrenière, D., Doyon, R., Macintosh, B., \& Nadeau, D. 2006, ApJ, 641, 556

Smith, B. A. \& Terrile, R. J. 1984, Science, 226, 1421 


\section{Discussion}

CURRIE: This is a very impressive system. I had two small detail-oriented questions. (1) What are the SNRs you're getting in the images? (2) Could you explain the shape/ structure of the subtracted PSF?

MorzInski: Because the VisAO and Clio images were obtained simultaneously, and we calibrated the astrometry of the two cameras, we are confident in all the detections presented. For your second question, the radial elongation and tangential negative flux are artifacts. PCA is trying to fit a high-spatial-frequency feature with low-order modes. Thus you get ringing just like in Fourier methods. I have not done any filtering or masking of the planet in the images. Therefore, this is a true image of the residuals after using PCA to subtract the PSF.

Bonnefoy: Very excellent talk. The instrumental strategy is amazing. Just to see these PSFs with the adaptive secondary and the pyramid wavefront sensor is so amazing. I just have a comment. I think that the new data points you are getting in the red optical [NOTE: see Males et al. 2013] and also the complementary data points are very interesting. I played with the metallicity with the BT Settl and the DRIFT-PHOENIX models and I remember that the $J$-band flux and the flux at shorter wavelengths is dependent on the metallicity, so you might be able to say more about the metallicity of the object.

MoRzInski: Thank for the compliment. That is very interesting, thank you very much.

KEnwORTHY: I was really amazed at the $1-\mu \mathrm{m} \beta$ Pic b image which is very spectacular [NOTE: see Males et al. 2013]. But I was wondering, what kind of astrometric precision are you getting?

Morzinski: Great question. People don't talk about this much, but one of the most difficult things in measuring astrometry of directly-imaged exoplanets is actually centroiding on the host star, which is saturated. Due to the fundamental nature of ADI-like methods, the center of the host star is also the center of rotation for the image processing - derotating and combining images - so this is why the PSF centration is the fundamental limit. We tried various techniques to centroid on the central star, and found that in the case of MagAO/Clio images, they are circularly symmetric enough such that we found the best center of rotation by minimizing the residuals of an image rotated by $\theta$ degrees differenced from the original image, where $\theta$ varies from 5 to 355 degrees in 10-degree increments. Centroiding to a tenth of a pixel precision still gives $\sim 10 \%$ error in the position of the planet. Although we used Trapezium observations to calibrate platescale and distortion, and to calibrate Clio and VisAO together, in fact we found that the limit to astrometric precision is set by how well we can determine the center of the host-star PSF. 